\title{
Inertial floaters in stratified turbulence
}

\author{
A. Sozza, F. De Lillo and G. Boffetta \\ Department of Physics and INFN, Università di Torino, via P. Giuria 1, 10125 Torino, Italy
}

\begin{abstract}
We investigate numerically the dynamics and statistic of inertial particles transported by stratified turbulence, in the case of particle density intermediate in the average density profile of the fluid. In these conditions, particles tend to form a thin layer around the corresponding fluid isopycnal. The thickness of the resulting layer is determined by a balance between buoyancy (which attracts the particle to the isopycnal) and inertia (which prevents them from following it exactly). By means of extensive numerical simulations, we explore the parameter space of the system and we find that in a range of parameters particles form fractal cluster within the layer.
\end{abstract}

\section{INTRODUCTION}

The interaction between particle inertia and turbulence is a fundamental issue for many problems in natural sciences and in applications, from cloud formation in the atmosphere to the dynamics of plankton in the ocean [1, 2]. Most of the studies have considered the simplified case of inertial particles in homogeneous-isotropic turbulence [3 5], while more recent works have investigated the interaction between gravity and turbulent acceleration [6, 7].

In the case of stratified turbulence, gravity plays two different roles: from one side it produces a vertical motion of inertial particles with respect to the flow which can increase sedimentation; on the other side it generates a layered structure in the flow by reducing the vertical velocity fluctuations and promoting bidimensionalization [8, 9].

The motion of inertial particles in stratified turbulence has been recently addressed by means of direct numerical simulations within the Boussinesq approximation of incompressible flow [10 12]. In particular it has been shown that inertial particles clusterize for a wide range of parameters in a simplified overdamped limit in which inertia feels the effect of gravity only. In that limit, it has been found that vertical confinement due to density stratification induces a dissipative dynamics of the particles and this produces a strong fractal clustering on the isopycnal surface. Fractal clustering depends on a single parameter, combination of the Stokes time of the particles and the Brunt-Väisälä frequency of the flow and is maximum for large Stokes numbers [12].

In this Letter we extend the previous investigation by considering the Maxey-Riley model for small inertial particles with finite inertia [13], similar to the model used in [10]. We confirm the vertical confinement of the particles induced by stratification and we explain the non monotonic dependence on the parameters by introducing a simple stochastic model for the vertical motion. Small-scale fractal clustering, quantified by the fractal dimension of particle distribution, also displays a nonmonotonic behavior, typical of inertial particles in incompressible flows, but with a peculiar scaling law for small
Stokes numbers due to the presence of density stratification.

In this paper we will study the behavior of a widely used model for inertial particles 13] in order to go beyond the limit of small inertia in the description of layer formation. First, we will describe the numerical model for the flow and the particles and introduce the physical parameters controlling the dynamics. Then, we will present the numerical results on the vertical distribution of the floaters and the small scale inhomogeneity. Finally, the results will be discussed in the conclusions.

\section{EQUATION OF MOTION FOR A FLOATER}

We consider a fluid linearly and stably stratified in the gravity direction $\mathbf{g}=(0,0,-g)$ with density profile $\rho=\rho_{0}-\gamma(z-\theta)$. Within the Boussinesq approximation the equations of motion for the incompressible velocity $\mathbf{u}$ (with $\nabla \cdot \mathbf{u}=\mathbf{0}$ ) and the density fluctuation $\theta$ are

$$
\begin{gathered}
\partial_{t} \mathbf{u}+\mathbf{u} \cdot \nabla \mathbf{u}=-\nabla p-N^{2} \theta \hat{\mathbf{z}}+\nu \nabla^{2} \mathbf{u}+\mathbf{f}, \\
\partial_{t} \theta+\mathbf{u} \cdot \nabla \theta=w+\kappa \nabla^{2} \theta
\end{gathered}
$$

where $\nu$ is the kinematic viscosity, $\kappa$ is the diffusivity and $N^{2}=\gamma g / \rho_{0}$ is the Brunt-Väisälä frequency. In the rhs, $\mathbf{f}$ represents an external forcing, which injects energy in the system at a rate $\varepsilon$ to maintain a stationary state, and is confined on a characteristic large-scale $\ell_{f}$. For simplicity and numerical convenience we only consider the case with Prandtl number $\operatorname{Pr}=\nu / \kappa=1$. Three dimensionless parameters can be defined in terms of the viscous scale $\eta=$ $\left(\nu^{3} / \epsilon\right)^{1 / 4}$, the Ozmidov scale $\ell_{0}=\left(\epsilon / N^{3}\right)^{1 / 2}$ and the forcing scale $\ell_{f}$ : the Reynolds number $R e=\left(\ell_{f} / \eta\right)^{4 / 3}$, the Froude Number $F r=\varepsilon^{1 / 3} \ell_{f}^{2 / 3} / N=\left(\ell_{0} / \ell_{f}\right)^{2 / 3}$ and the buoyancy Reynolds number $\operatorname{Re}_{b}=F r^{2} R e=\left(\ell_{0} / \eta\right)^{4 / 3}$ [9].

Particles are assumed to be spherical with a density equal to the reference density $\rho_{0}$ of the fluid. This assumption simplifies the notation without loss in generality, since the flow is homogeneous. We also assume that their radius $a$ and their velocity relative to the fluid, $\mathbf{v}-\mathbf{u}$, 

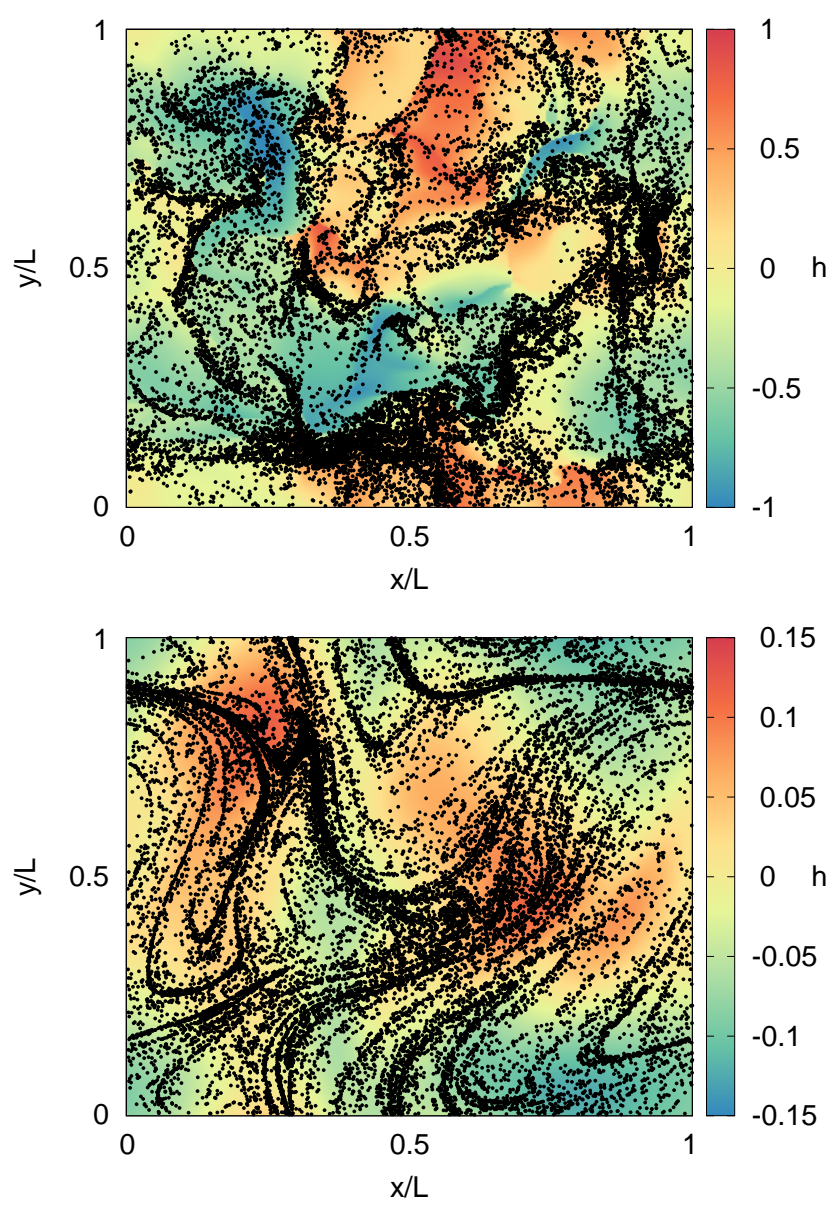

FIG. 1. (Color online) Snapshots of the distribution of inertial floaters with $S t=2$ over the isopycnal height $h$, implicitly defined by the equation $z-\theta=0$ for simulations with $\mathrm{Fr}=$ 0.4 (top panel) and $F r=0.08$ (bottom panel). Resolution $M=256$.

are small enough to guarantee creeping flow conditions around the particle, i.e. with particle's Reynolds number $R e_{p}=|\mathbf{v}-\mathbf{u}| a / \nu \ll 1$. Consistently with these assumptions, particle position $\mathbf{x}$ and velocity $\mathbf{v}$ are governed by a simplified version of the Maxey-Riley equation for spherical inertial particles [13], as

$$
\begin{gathered}
\dot{\mathbf{x}}=\mathbf{v}, \\
\dot{\mathbf{v}}=\beta \frac{D \mathbf{u}}{D t}-\frac{\mathbf{v}-\mathbf{u}}{\tau_{p}}+(1-\beta) \mathbf{g} .
\end{gathered}
$$

The added mass term in eq. (4) is proportional to the time derivative along fluid trajectories (denoted by the material derivative $D / D t)$ of the fluid velocity $\mathbf{u}(\mathbf{x}, t)$ at the position of the particle. The parameter $\beta=$ $3 \rho /\left(\rho+2 \rho_{0}\right)$ is a function of the ratio between the particle's density $\rho_{0}$ and the local density $\rho$ of the surrounding fluid, while $\tau_{p}=a^{2} /(3 \nu \beta)$ is the Stokes time. We remark
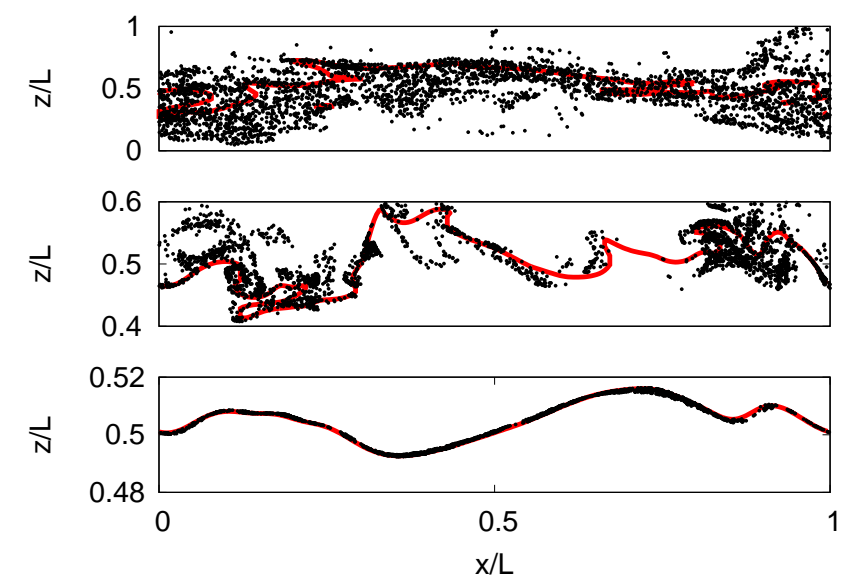

FIG. 2. (Color online) Snapshots of the vertical distribution of inertial floaters with $S t=2$ for increasing stratification strength (from top to bottom $\mathrm{Fr}=0.8,0.4,0.08$ ). Resolution $M=256$.

that in eq. (44) we neglect the so-called Faxen's correction and the Basset's history term since they give a negligible contribution for small particles. Taking into account the expression for the density profile, we can write $(1-\beta) g \simeq \frac{2}{3} N^{2}(z-\theta)$. Consistently with the Boussinesq approximation, we further neglect the term $(1-\beta)$ when not multiplied by gravity acceleration to obtain the equation for particle velocity

$$
\dot{\mathbf{v}}=\frac{D \mathbf{u}}{D t}-\frac{\mathbf{v}-\mathbf{u}}{\tau_{p}}-\frac{2}{3} N^{2}(z-\theta) \hat{\mathbf{z}}
$$

The dimensionless parameter which measures particle inertia is the Stokes number $S t=\tau_{p} / \tau_{\eta}$ where $\tau_{\eta}$ is the Kolmogorov time-scale of the flow.

\section{NUMERICAL SIMULATION}

We have performed direct numerical simulations of the Boussinesq equation for a stratified flow eqs.(112) in a periodic box of size $L=2 \pi$ by using a fully-dealiased pseudo-spectral method in space with a second-order Runge-Kutta scheme for time evolution. Turbulence is maintained in a statistical steady state by a random $\delta$ correlated in time forcing acting at large-scale in a narrow band of wavenumbers peaked at $k_{f} \simeq 1 / \ell_{f}$. We have used different spatial resolutions $M=128,256$, corresponding to Reynolds numbers $R e=\left(k_{f} \eta\right)^{-4 / 3} \sim 390,930$ respectively. We have considered 4 different values of stratification with Brunt-Väisälä frequencies corresponding to Froude numbers $\mathrm{Fr}=0.8,0.6,0.16,0.08$ and buoyancy Reynolds numbers $R e_{b}$ in the range $0.2-35$. We remark that for this flow the energy is composed by a kinetic and a potential contributions and therefore the viscous 
energy dissipation rate is typically smaller than the energy input rate $\varepsilon$ since a fraction of the kinetic energy is converted into potential energy and removed from the system through the cascade of potential energy [14]. As a consequence, small-scale parameters $\eta$ and $\tau_{\eta}$ have a small dependence upon stratification.

Once the turbulent flow has reached a statistical steady state, we introduce 20 populations of $M_{p}=1.6 \times 10^{5}$ inertial tracers each, characterized by different $S t$ numbers between $S t=0.01$ and $S t=100$. The initial distribution for each population is uniform in the domain and their trajectories are evolved according to eqs.(3) (5).

Figure plots two snapshots of the particle distribution (for $S t=2.0$ ) together with the isopycnal surface $h$, implicitly defined by the equation $z=\theta(\mathbf{x}, \mathbf{t})$ for two values of stratification $F r=0.4$ (top panel) and $F r=0.08$ (bottom panel). We observe that particles distribution is correlated with the interfacial regions between positive and negative values of $\theta$. Such a correlation has been observed previously for inertial particles in homogeneous isotropic turbulence [15]. Indeed, once floaters have reached their isopycnal surface, they are constrained to perform a quasi-horizontal motion which is unaffected by gravity and resembles that of the inertial particles accumulating at the fronts.

The vertical distribution of particles is shown in fig. 2 for different values of stratification of the velocity field. The vertical confinement is produced by the last term in eq. (5) which is proportional to $N^{2}$, and it is therefore strongly dependent on stratification. For the most stratified case, $F r=0.08$, particles are practically confined on the isopycnal surface $z=\theta$, which correspond to the density $\rho=\rho_{0}$.

\section{Statistics of vertical confinement}

To better understand the mechanism of layer formation, we can first consider the limit of small St. For $\tau_{p} \rightarrow 0$, we can take the overdamped limit of eq. (5) which, after defining $\tau=3 /\left(2 N^{2} \tau_{p}\right)$ becomes

$$
\dot{\mathbf{x}}=\mathbf{u}-\frac{1}{\tau}(z-\theta) \hat{\mathbf{z}} .
$$

The comparison with eq. (2) shows that in this limit, neglecting diffusion, the quantity $(z-\theta)$ exponentially relaxes to zero with the characteristic time $\tau$. Our numerical simulations confirm this picture, and the variance of the vertical position $\sigma_{z}^{2}=\left\langle z^{2}\right\rangle-\langle z\rangle^{2}$ (the brackets indicate Lagrangian average) decreases from the initial value $\sigma_{0}^{2}=\pi^{2} / 3$ (corresponding to uniform vertical distribution) and reaches a stationary value after a time of order $\tau$. In the limit of small $S t$, particles distribution is almost homogeneous (with $\sigma_{z}=\sigma_{0}$ ), while for increasing $S t, \sigma_{z}$ drops down indicating that a layer of thickness smaller than the box length is formed.

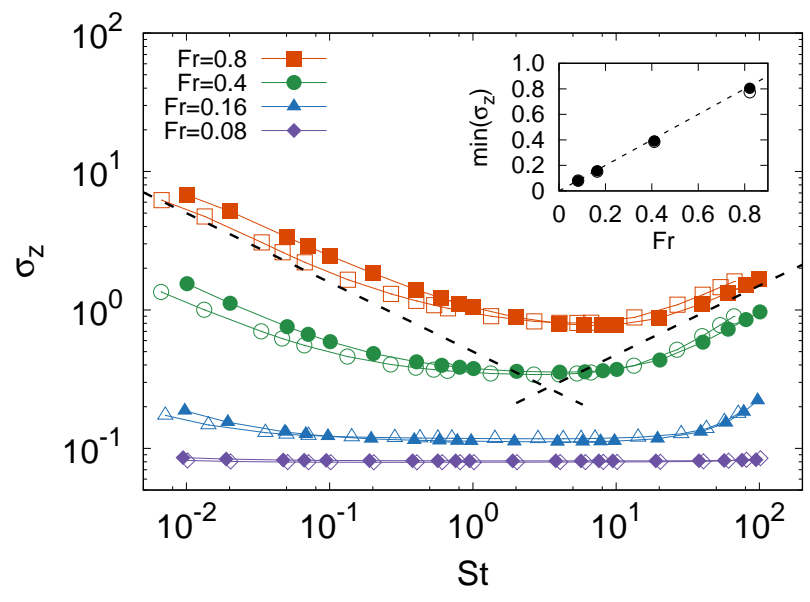

FIG. 3. (Color online) Layer thickness $\sigma_{z}$ as a function of $S t$ for different stratification $F r=0.8$ (red squares), $F r=$ 0.4 (green circles), $F r=0.16$ (blue triangles), $F r=0.08$ (purple diamonds) and resolution $M=128$ (empty symbols) and $M=256$ (filled symbols). Dashed lines represents the behavior $S t^{1 / 2}$ and $S t^{-1 / 2}$. Inset: Behavior of $\sigma_{z}$ in $F r$ in the case of maximum confinement.

The stationary root mean square of the vertical position as a function of the Stokes number and for different Froude numbers is shown in fig. which confirms the qualitative observation of fig. 2. vertical confinement strongly depends on stratification, following the fluctuations of the isopycnal surface $z-\theta=0$. For each $F r$, we find a maximum confinement for intermediate values of the Stokes numbers, $S t=O(1)$. This behavior can be understood since in the limit of small $S t$ the last term in eq. (6) is small ( $\tau$ is large) and particle trajectory is close to that of a fluid particle. In the opposite limit of large $S t$, particles are unable to follow the rapid fluctuations of the isopycnal surface and this produces a thick layer around the isopycnal surface.

The above argument can be made more quantitative by considering the stochastic version of eq. (5) in which the fields $\mathbf{u}$ and $\theta$ are Gaussian noises with zero mean and variances $2 D_{u}$ and $2 D_{\theta}$. The vertical motion of the floaters is therefore governed by an Ornstein-Uhlenbeck process for the probability $p(z, v)$ to find a particle at the vertical position $z$ with a vertical velocity $v$. The associated Fokker-Planck equation is [16]

$$
\partial_{t} p+\partial_{z}(v p)-\partial_{v}\left[\left(\frac{v}{\tau_{p}}-\frac{2 N^{2}}{3} z\right) p\right]=D \partial_{v}^{2} p
$$

which has as solution a Gaussian distribution. The marginal distribution of particle positions is also Gaussian with variance $\sigma_{z}^{2}=\frac{3 \tau_{p}}{2 N^{2}} D$, where

$$
D=\frac{1}{\tau_{p}^{2}} D_{w}+\frac{4}{9} N^{4} D_{\theta}
$$



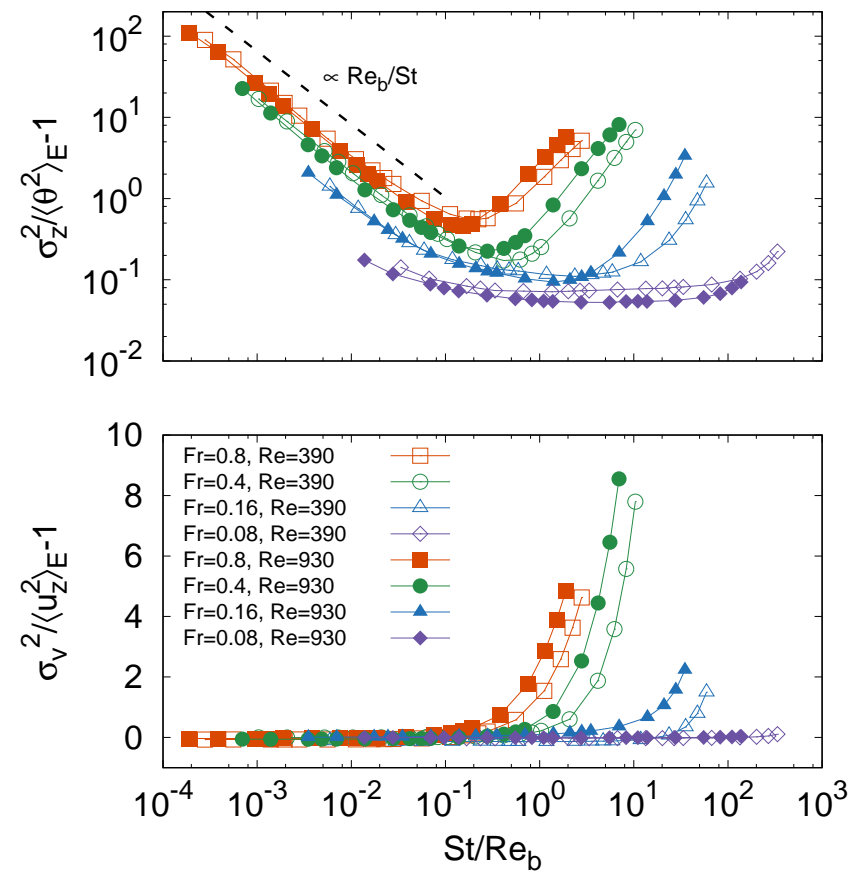

FIG. 4. (Color online) Variance of the layer thickness $\sigma_{z}^{2}$ normalized with the Eulerian-averaged variance of the fluctuation density field $\left\langle\theta^{2}\right\rangle$ (top panel) and variance of the vertical particle velocity $\sigma_{v}^{2}$ normalized with the Eulerian-averaged variance of the vertical fluid velocity $\left\langle u_{z}^{2}\right\rangle$ (bottom panel) as a function of $S t / R e_{b}$ for different stratification $F r=0.8$ (red squares), $F r=0.4$ (green circles), $F r=0.16$ (blue triangles), $F r=0.08$ (purple diamonds) and resolutions $M=128$ (empty symbols) and $M=256$ (filled symbols).

is the total diffusivity due to $D_{w}$, the eddy diffusivity due to the fluctuating turbulent velocity, and $D_{\theta}$, the diffusivity due to the fluctuations of the stratification (we neglect the contribution in $D$ due to the added mass since it does not change the following argument). The expression eq. (8) explains the non-monotonic behavior observed in fig. . Indeed for small $\tau_{p}$ the first term in the r.h.s. of eq. (8) dominates and we get the behavior $\sigma_{z}^{2} \simeq$ $3 D_{w} /\left(2 N^{2} \tau_{p}\right) \propto R e_{b} S t^{-1}$ (also observed in [17]), while in the opposite limit we get $\sigma_{z}^{2} \simeq 2 D_{\theta} N^{2} \tau_{p} / 3 \propto R e_{b}^{-1} S t$.

Figure shows that $\sigma_{z}$ strongly depends on the stratification, since for small $\mathrm{Fr}$ the isopycnal surface to which particles are attracted is more confined, as shown in fig. 2. This can be also understood from the stochastic model eqs. (7, in the limit of small $S t$ since there we have $\sigma_{z} \propto 1 / N$ which is indeed close to the numerical results as shown in the inset of fig. which confirms previous results obtained within the overdamped model [12]. We observe that in the opposite limit of large $S t, \sigma_{z}$ contains the diffusivity $D_{\theta}$ which is itself dependent on $N$.

In fig., the variance $\sigma_{z}^{2}$ as a function of $S t / R e_{b}$ is shown for different stratifications. For small values $S t / R e_{b}$, nu- merical results confirm the prediction of the stochastic model.

\section{Statistics of vertical particle velocity}

A similar argument using the Fokker-Planck equation eq. (6) can be exploited to study the vertical particle velocity $v$. As before, the marginal distribution of particle velocity is Gaussian with variance given by $\sigma_{v}^{2}=D_{w} \tau_{p}^{-1}+\frac{4}{9} N^{4} \tau_{p} D_{\theta}$. In fig. (bottom panel), the vertical particle velocity $v$ is observed to increase with $S t$. In the limit of small inertia, the particle velocity has to coincide with the velocity of the surrounding fluid. In the opposite limit, when $S t \gg 1$ the vertical particle velocity slightly differs from the fluid velocity and it is found to increase approximately as $S t^{1 / 2}$. This is very different from the case of inertial particles, for which it has been observed that particle velocity decreases as $S t^{-1 / 2}$ [18].

\section{Small-scale fractal clustering}

In addition to the vertical, large scale inhomogeneity due to layering around the equilibrium isopycnal, particle dynamics induces small-scale clustering within the layer, which is evident from the horizontal section shown in fig. . This small-scale clustering is due to the dissipative dynamics eq. (5) which governs the motion of the tracers and it is typical of inertial particles in incompressible turbulence. As a consequence, trajectories in phase-space converge onto a dynamic attractor of smaller dimension and, when the attractor has dimension smaller than the space dimension $d=3$, particles distribute on a fractal set of the same dimension [5].

In order to characterize small-scale clustering we computed the correlation dimension $D_{2}$ of the particle distribution, defined through the probability $p_{2}(r) \sim r^{D_{2}}$ of finding two particles within a distance $r$. A homogeneous distribution in a d-dimensional space would clearly give $D_{2}=d$. Figure shows $p_{2}(r)$ for different values of $S t$ and Fr. A clear scaling range can be observed between $0.1 \eta-10 \eta$, from which one obtains a measure of the correlation dimension $D_{2}$. The correlation dimension as a function of $S t$ and for various values of $F r$ is plotted in the inset. In all cases, the strongest clustering is observed for intermediate values of $S t$, while extreme (small or large) values of $S t$ produce more homogeneous layers. Similar phenomenology is also observed in the small scale clustering of inertial particles also in homogeneous, isotropic turbulence [5].

One can focus more precisely on the deviations from homogeneity (for which $D_{2}=3$ ) by considering the codimension $3-D_{2}$, which is plotted in fig. for the same values of $\mathrm{Fr}$. This figure clearly shows a power-law increase of the co-dimension with $S t$ in the limit of low 


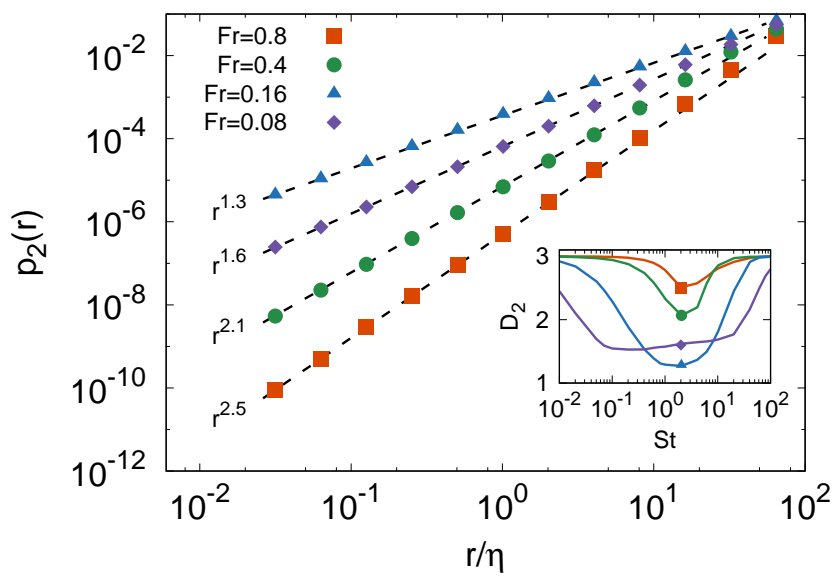

FIG. 5. Correlation integral $p_{2}(r)$ as a function of $r / \eta$ for different values of stratification $F r=0.8$ (red squares), $F r=$ 0.4 (green circles), $F r=0.16$ (blue triangles), $F r=0.08$ (purple diamonds)..and Stokes Number $S t=2$. Resolution $M=256$. Inset: Correlation dimension as a function of $S t$ for different stratification.

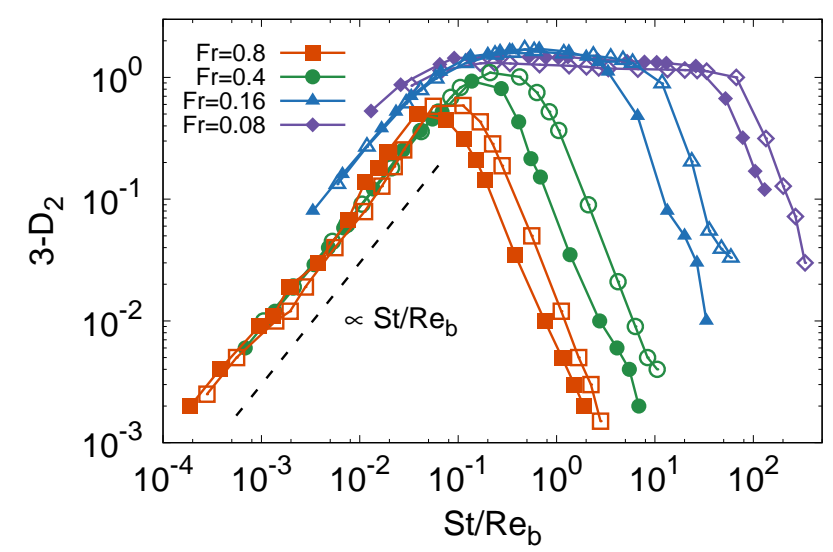

FIG. 6. Co-dimension $3-D_{2}$ as a function of $R=\frac{2}{3} R e_{b} S t^{-1}$ for different stratification $F r=0.8$ (red squares), $F r=0.4$ (green circles), $F r=0.16$ (blue triangles), $F r=0.08$ (purple diamonds) and resolutions $M=128$ (empty symbols) and $M=256$ (filled symbols). Dashed line represents the prediction $3-D_{2} \propto R^{-1}$.

inertia.

However, comparison between fig. and analogous measurements in the literature on inertial particles in isotropic turbulence show a striking difference in the small-St behavior of the curves. In the case where buoyancy is neglected one can show that $3-D_{2} \sim S t^{2}$ for $S t \rightarrow 0$ while in our case $3-D_{2} \sim S t$ in the limit of small particles, as one can see in fig. . This behavior can be rationalized following [4, 19, 20]. In the small $S t$ limit, one can again use the approximation of eq. (6), in which the particles are advected by an effective velocity field $\mathbf{v}(\mathbf{x}, t)=\mathbf{u}-(1 / \tau)(z-\theta) \hat{\mathbf{z}}$. The number density of the particles evolves as $d n / d t=-(\nabla \cdot \mathbf{v}) n=\left(1-\partial_{z} \theta\right) / \tau$. The particle distribution will be homogeneous above a certain scale $\ell \gtrsim \eta$, with a constant number density $n_{0}$, and fractal on smaller scales due to the chaotic dynamics. If $n$ is sampled with boxes of size $r \ll \ell$, the density fluctuations measured will be those accumulated along a Lagrangian trajectory during a time $T_{r}=\ln (\ell / r) /\left|\lambda_{3}\right|$, i.e. the time it takes for chaotic advection to compress the initial patch along the direction characterized by the most negative Lyapunov exponent $\lambda_{3}$. One therefore has $n^{2} \sim n_{0}^{2} \exp \left[2 \int_{0}^{T_{r}} \tau^{-1}\left(1-\partial_{z} \theta\right) d t\right]$. Eulerian average requires to weigh each patch with its volume, which contracts as $1 / n$ so one has

$$
\left\langle n^{2}\right\rangle=n_{0}^{2}\left\langle\exp \left[\int_{0}^{T_{r}} \tau^{-1}\left(1-\partial_{z} \theta\right) d t\right]\right\rangle \sim\left(\frac{r}{\eta}\right)^{\alpha}
$$

If we assume the integral to be dominated by the constant term we get $\alpha=1 /\left(\left|\lambda_{3}\right| \tau\right)$. Of course this requires that $\left|\partial_{z} \theta\right| \ll 1$, i.e. that the density fluctuations are small. This condition is typical for $R e_{b}=O(1)$ or smaller, while it is clearly violated around folds in the isopycnal. For small $S t$ it is reasonable to assume that $\lambda_{3} \propto \tau_{\eta}^{-1}+O(1 / \tau)$, so given the definition of the correlation dimension, $3-D_{2}=\alpha \simeq \tau_{\eta} / \tau \simeq R e_{b}^{-1} S t$.

It may be interesting to notice that the behavior of $D_{2}$ is similar to that of $\left\langle\sigma_{z}\right\rangle$ in fig. . In particular, both observables have a minimum near $S t \sim O(1)$. Moreover, both quantities shows a plateau in the region of maximum clustering, which becomes more evident when the fluid is intensely stratified.

Indeed, if $R e_{b}$ is very small, isopycnals are very close to flat surfaces and vertical fluctuations are suppressed.

\section{CONCLUSIONS}

We investigated the behavior of inertial particles advected by stratified turbulence, in the case of particle density intermediate within the fluid density gradient. We have studied how the interplay of gravity and turbulence produces a vertical confinement of the inertial particles around the isopycnal surface at the particle density, and how the resulting dissipative dynamics produces fractal clustering at small scales.

The interaction between particle settling and turbulence is an essential problem for many systems in the natural sciences and in applications. In marine and lake biology, the possibility of organisms to control their position in the water column is of great importance for the uptake of nutrients, the access to light and for escaping predators 21, 22]. In the absence of specific mechanisms, the survival of such organisms must result from the complex 
interplay between turbulent mixing and growth [23, 24]. A particularly interesting problem is posed by thin phytoplankton layers (TPL), aggregations of phytoplankton and zooplankton at high concentration, with thickness from centimeters to few meters, extending up to several kilometers horizontally and with a time scale from hours to days [2]. Various mechanisms have been proposed to explain such formations, notably depending on the motility (or absence thereof) of a particular species observed to form TPLs. In particular, If a cell is neutrally buoyant at some intermediate depth in the pycnocline, then buoyancy could lead to the formation TPLs [2, 12, 25].

The physical and biological implications of fractal clustering observed at small scales are associated with an increased probability to find particles at small separations with respect to a homogeneous distribution. Numerical studies inspired by TPLs showed that this can have important effects on the population dynamics of plankton [26]. In general, small scale clustering is relevant in problems where encounter rates are important, such as mating and competition for resources in biology, coalescence and coagulation of droplets in cloud physics [19, 27] and engineering applications [28].

We thank M. Cencini for useful discussions. We acknowledge support from the COST Action MP1305 "Flowing Matter" and from Cineca within the INFNCineca agreement INF17turb.

[1] W. Grabowski and L.-P. Wang, Annual Review of Fluid Mechanics 45, 293 (2013).

[2] W. Durham and R. Stocker, Annual review of marine science 4, 177 (2012).

[3] K. Squires and J. Eaton, Physics of Fluids A: Fluid Dynamics 3, 1169 (1991).

[4] E. Balkovsky, G. Falkovich, and A. Fouxon, Physical Review Letters 86, 2790 (2001).

[5] J. Bec, Physics of fluids 15, L81 (2003).

[6] J. Bec, H. Homann, and S. Ray, Physical review letters
112, 184501 (2014).

[7] K. Gustavsson, S. Vajedi, and B. Mehlig, Phys. Rev. Lett. 112, 214501 (2014).

[8] J. Riley and M.-P. Lelong, Annual review of fluid mechanics 32, 613 (2000).

[9] G. Brethouwer, P. Billant, E. Lindborg, and J.-M. Chomaz, Journal of Fluid Mechanics 585, 343 (2007).

[10] M. Van Aartrijk and H. Clercx, Physical review letters 100, 254501 (2008).

[11] M. Van Aartrijk and H. Clercx, Physics of Fluids 22, 013301 (2010).

[12] A. Sozza, F. De Lillo, S. Musacchio, and G. Boffetta, Physical Review Fluids 1, 052401 (2016).

[13] M. Maxey and J. Riley, The Physics of Fluids 26, 883 (1983).

[14] A. Sozza, G. Boffetta, P. Muratore-Ginanneschi, and S. Musacchio, Physics of Fluids 27, 035112 (2015).

[15] J. Bec, H. Homann, and G. Krstulovic, Physical review letters 112, 234503 (2014).

[16] C. Gardiner, Stochastic methods (Springer Series in Synergetics, 1985).

[17] D. A. Birch, W. Young, and P. Franks, Limnology and Oceanography 54, 397 (2009).

[18] J. Abrahamson, Chemical Engineering Science 30, 1371 (1975).

[19] G. Falkovich, A. Fouxon, and M. Stepanov, Nature 419, 151 (2002).

[20] I. Fouxon, Physical review letters 108, 134502 (2012).

[21] C. Reynolds, The ecology of phytoplankton (Cambridge University Press, 2006).

[22] T. Kiørboe, A mechanistic approach to plankton ecology (Princeton University Press, 2008).

[23] J. Huisman, M. Arrayás, U. Ebert, and B. Sommeijer, The American Naturalist 159, 245 (2002).

[24] R. Margalef, Oceanologica acta 1, 493 (1978).

[25] A. Alldredge, T. Cowles, S. MacIntyre, J. Rines, P. Donaghay, C. Greenlaw, D. Holliday, J. Margaret M. Dekshenieks, and J. Zaneveld, Mar Ecol Prog Ser 233, 1 (2002).

[26] P. Perlekar, R. Benzi, D. R. Nelson, and F. Toschi, Physical review letters 105, 144501 (2010).

[27] R. A. Shaw, Annual Review of Fluid Mechanics 35, 183 (2003).

[28] S. L. Post and J. Abraham, International Journal of Multiphase Flow 28, 997 (2002). 